\title{
ON THE CONTROLLABILITY OF THE BURGER EQUATION
}

\author{
T. HORSIN
}

\begin{abstract}
We present here a return method to describe some attainable sets on an interval of the classical Burger equation by means of the variation of the domain.
\end{abstract}

\section{Statement of THE MAin RESUlt}

We are here interested in the following problem of controllability: Let $T_{c}$ be an arbitrary real number, $X$ a given normed space of real functions of the real variable defined on $[1,2], z_{1}, z_{0}$ elements of $X$. Does there exist a weak (entropic) solution of:

$$
\begin{gathered}
u: \mathbb{R}^{+} \times \mathbb{R} \rightarrow \mathbb{R} \\
u \in L^{\infty}((0,+\infty), X), \\
u_{t}+\left(\frac{u^{2}}{2}\right)_{x}=0, \\
u(0, .) /[1,2]=z_{0}, \\
u\left(T_{c}, .\right) /[1,2]=z_{1} ?
\end{gathered}
$$

If this holds for all $\left(z_{0}, z_{1}\right) \in X \times X$ one says that equation (1.2) is controllable in $X$. Moreover if one replaces condition (1.4) by

$$
\left\|u\left(T_{c}, .\right) /[1,2]-z_{1}\right\|_{X}<\varepsilon
$$

where $\varepsilon>0$ is a priori given, and if this holds for any $\left(z_{0}, z_{1}\right) \in X \times X$ and any $\varepsilon>0$, one says that there is approximate controllability in $X$. According to the settlement of the controllability problem, we take here $X=B V([1,2])$ equipped with the $L^{1}$ norm. If $A \subset \mathbb{R}, B V(A)$ denotes the space of mesurable functions $f$ such that

$$
\int_{A}|f| d x+\int|D f|<+\infty
$$

here $D f$ is the derivative of $f$ in the sense of measures.

The following conditions (ES) are the conditions necessarily satisfied by spatial values of weak entropic solutions of the Cauchy problem (1.2) with initial value in the class of functions with bounded variation: we say that a

Université de Versailles, Analyse appliquée, 78030 Versailles Cedex, France and CMLA, ENS-Cachan, 94235 Cachan Cedex, France. E-mail: horsin@fermat.math.uvsq.fr.

Received by the journal February 27, 1997. Revised September 17, 1997. Accepted for publication March 12, 1998.

(C) Société de Mathématiques Appliquées et Industrielles. Typeset by IATEX. 
function $f$ satisfies (ES), and is said to be of entropic shape, if

$$
\begin{gathered}
f \in B V \\
\forall x, f(x-):=\lim _{t \rightarrow x, t<x} f(t) \geq f(x+):=\lim _{t \rightarrow x, t>x} f(t),
\end{gathered}
$$

(1.6) is strict for at most a sequence $\left(x_{\nu}\right)$

The main proposed result in this paper is the following:

Theorem 1.1. Let $z_{0}$ be given in $B V([1,2]) \cap L^{\infty}([1,2])$ and $\varepsilon>0$ and $T>2$. Let $z_{1}$ be an element of $B V([1,2])$ satisfying the necessary conditions (ES) and, such that

$$
\begin{aligned}
& \text { (i): } \forall x_{0} \in(1,2) \text {, if } z_{1}\left(x_{0}-\right)>0 \text { then } z_{1}(x)>\frac{x-1}{T} \forall 1 \leq x<x_{0} \text {, if } \\
& z_{1}\left(x_{0}+\right)<0 \text {, there holds } z_{1}(x)<\frac{x-2}{T} \forall x_{0}<x \leq 2,
\end{aligned}
$$

(ii): There exists at most one $x_{1} \in(1,2)$ such that $z_{1}>0$ on $\left[1, x_{1}\right)$ and $z_{1}<0$ on $\left(x_{1}, 2\right]$ : moreover for any $x \in(1,2]$ being a point of continuity of $z_{1}$ such that $z_{1}(x) \neq 0$ one has

$$
D^{+} z_{1}(x) \leq \frac{1}{T},
$$

then there exists a time $T_{c} \geq T$ ( $T_{c}$ is the time of approximate controllability) such that the equation (1.2) has at least a weak solution such that (1.3) and (1.4) hold if (1.4) is replaced by the following: there exists an interval of length $2 \varepsilon$ outside of which $u\left(T_{c},.\right)$ and $z_{1}$ are equal and moreover

$$
\int_{1}^{2}\left|u\left(T_{c}, x\right)-z_{1}(x)\right| d x \leq C\left(u_{0}, u_{1}\right) \varepsilon .
$$

This last statement is a little bit more than $L^{1}$ approximate controllability. Here $D^{+} z_{1}(x)$ means

$$
\sup _{h>0} \frac{z_{1}(x+h)-z_{1}(x)}{h} .
$$

To our knowledge, the problem of the controllability of the equation (1.2) has been initially studied in [1]. The major difference between [1] and the present work relies on the fact that the problem considered in [1] concerns only the solution on a half-plane (see [13], or [10]), and only for vanishing initial data. Nevertheless the method that we present here is of quite different nature, though, of course, it relies on the characteristic method. It has been inspired by the finite dimensional case in the theory of control.

Let us stress that (i) is given in [1]. Condition (ii) has also an equivalent in [1], which is

$$
D^{+} z_{1}(x) \leq \frac{z_{1}}{x-1}
$$

written in our situation, but (ii) requires more.

Let us also mention that for the Burger equation with addition of a diffusion term, a negative answer to a problem of partial boundary controllability was given by A. Fursikov \& O.Yu. Imanuvilov, see [8] and (it seems previously) by J.I. Diaz, see [5].

It is well known, see [14] or [15], that the space of bounded variation functions is a good regularity space for the Cauchy problem (1.2) \& (1.3) in the class of entropic solution. One also knows that $\forall t \in(0,+\infty) u(t,$. ESAIM: COCV, APRIL 1998, VOL. 3, 83-95 
has compact support (provided that the initial value has compact support) and is continuous except on a countable set of points being the boundary of a dense union of open intervals. At each discontinuity $x$ then the entropic shape of the solution (1.6) gives that

$$
u(t, x-)>u(t, x+) .
$$

According to these remarks, the conditions (ES) are necessary for $z_{1}$ to be attainable. In particular $z_{1}$ is continuous on the countable union of open intervals. The standard ideas on the Cauchy problem (1.2) \& (1.3) can be found in [11] and in [12]. We also recall, see [18], that standard rules of differentiation can be used in the space $B V$ provided that one replaces the functions by some regularization. In particular, if $f \in B V$ and if one defines $\tilde{f}(x)=\frac{1}{2}(f(x+)+f(x-))$ one has the formula $\left(f^{2}\right)_{x}=2 \tilde{f} f_{x}$.

\section{Proof of the RESUlT}

A natural idea to show the controllability (at least locally) is to deal with the linearized operator around the null solution. This method, a classical one for finite dimensional systems, gives also many results for partial differential equations, even if it is harder to proceed in that case, as it has been shown recently by A. Fursikov and O.Yu. Imanuvilov, see [8] and L. Rosier [16]. This trick, astutely rethought, gives also global results, when the linearized operators around any trajectories are controllable, as it was proven by $\mathrm{C}$. Fabre \& J-P. Puel \& E. Zuazua in [6] \& [7].

Unfortunately, for the equation (1.2), the linearized operator around 0 is

$$
z_{t}=0 \text { on }[1,2]
$$

which is not even approximately controllable.

In order to obtain our result we have been interested in the so called "return method" introduced by J-M. Coron in [2] to show a result of stabilization of a finite dimensional system. He used it afterwards in [3] and [4] to study the controllability of incompressible fluids in dimension 2 . The idea is to consider the controllability of the linearized operator, not around the zero solution, but around trajectories vanishing at the times 0 and $T$ : if this linearized operator is controllable, one may hope the nonlinear system is locally controllable.

Of course such an entropic trajectory of (1.2) does not exist except if it is 0 on $[0, T] \times[1,2]$, and the linearized operator around the zero solution is not even approximately controllable, since any solution of the linearized equation is of course a constant of time.

Moreover, if one rescales such a solution (or more exactly an approximation), the time to attain the zero value is scaled too, thus one cannot expect to apply correctly the implicit function theorem, the essential reason being that for asymptotic large time a small difference between two solutions becomes very large, due to the term $u u_{x}$ in (1.2). 
However it is possible to provide solutions allowing to perform some kind of linearization as will be described later. Let us mention that the method used here relies more on a variation of the domain than on a standard linearization and our proof can also be related to the "extension method" introduced by D.L. Russell, see [17]. The control in our case is the initial value and can be realized by means of boundary values when it has some sense for (1.2).

In order to show Theorem 1.1, we first prove:

TheOREM 2.1. If $z_{0}=0, T>2$ be given, there exists a solution to (1.2), (1.3), (1.4), (2.2) provided that $z_{1}$ satisfies (ES) and conditions (i) and (ii) of Theorem 1.1. Moreover we can choose $T_{c}=T$.

The proofs of Theorem 1.1 and Theorem 2.1 are organized as follows: In 2.1 we give the necessary condition for $z_{1}$ to be attainable, then in 2.2 , we describe the sufficient conditions that lead to the conclusion of those theorems.

\subsection{NECESSARY CONDITIONS FOR CONTROLLABILITY}

We will now assume that $T>2$. Since the problem we are concerned with in proposition 2.3, requires that the initial value of $u$ must be 0 on $[1,2]$ whatever is $z_{1}$, we see that it is necessary to impose

$$
\forall y \in[1,2], z_{1}(y) \geq \frac{y-1}{T}\left(\text { or } z_{1}(y) \leq \frac{y-2}{T}\right) .
$$

We will treat the first case since the second is similar. Now assume that for some $\bar{y} \in[1,2]$ equality holds, then in order for $z_{1}$ to be attained by the Cauchy problem, we have

Assertion 2.2. For $y>\bar{y}$ equality holds in (2.1).

Proof: If there exists $y>\bar{y}$ such that inequality holds, then the generalized backward characteristics issued from the point $(\bar{y}, T)$ and the point $(y, T)$ would meet on $(0, T)$ contradicting the assumption on the entropic shape of $z_{1}$. So equality holds which proves this assertion.

Now by the method of characteristics one immediately sees that such a $z_{1}$ can only be attained by imposing a Dirac mass at time $t=0$ for $x=1$, since $z_{0 /[1,2]}=0$, and thus it is not reachable by our method. Thus the only possibility for equality is $\bar{y}=2$ but this also leads to the same conclusion.

So we found one necessary controllability condition for $z_{1}$ (in the present case) which is $z_{1}(x)>\frac{x-1}{T}$. Let us stress that condition (2.1) does not depend on the device introduced here.

\subsection{Proof of the Sufficient Conditions For CONTRollability}

2.2.1. Proof of Theorem 2.1: case of continuous final state. We first of all assume stronger conditions.

Proposition 2.3. Assume moreover that $z_{1}>0$ on $[1,2]$. Then the result of Theorem 2.1 is true.

PROOF OF PROPOSITION 2.3

ESAIM: COCV, APRIL 1998, VoL. 3, 83-95 
We fix once for all a real nonnegative small number $\frac{1}{2}>\rho>0$. Let $a_{1, \rho}$ be the unique entropic solution of (1.2) defined by

$$
\begin{array}{cl}
a_{1, \rho}(x, 0)=0, & x \leq 0, \\
a_{1, \rho}(x, t)=\min \left(\frac{x}{t+\rho}, 1\right), & 0 \leq x \leq 1, \\
a_{1, \rho}(x, t)=0, & x \geq 1 .
\end{array}
$$

Standard computations using the Rankin-Hugoniot condition show that

$$
a_{1, \rho}(x, t)=\frac{x}{t+\rho} \cdot \chi_{x \leq \sqrt{(2-\rho) \cdot(t+\rho)}}, t \geq 2-2 \rho .
$$

Here $\chi$ means the characteristic function of the set in subscript.

Assume that $z_{1}$ is continuous. We look for solutions of (1.2) of the form (for sake of simplicity we forget the subscript 1 for the moment being in $\left.a_{1, \rho}\right)$.

$$
u(x, t)=\alpha a_{\rho}\left(\frac{x+\tau(x, t)}{\alpha}, t\right),
$$

where $\tau$ is of course to be precised and where we take $\alpha>0$. This is precisely the variation of the domain. Assume that the standard rule of differentiation of composed functions is valid, then if we put (2.2) into (1.2) it gives

$$
\begin{gathered}
\alpha \partial_{t} a_{\rho}\left(\frac{x+\tau(x, t)}{\alpha}, t\right)+\partial_{x} a_{\rho}\left(\frac{x+\tau(x, t)}{\alpha}, t\right) \partial_{t} \tau(x, t)+ \\
\alpha a_{\rho}\left(\frac{x+\tau(x, t)}{\alpha}, t\right) \partial_{x} a_{\rho}\left(\frac{x+\tau(x, t)}{\alpha}, t\right) .\left(1+\partial_{x} \tau(x, t)\right)=0 .
\end{gathered}
$$

Thus, if

$$
\partial_{t} \tau(x, t)+\alpha a_{\rho}\left(\frac{x+\tau(x, t)}{\alpha}, t\right) \partial_{x} \tau(x, t)=0,
$$

the function $u$ will be, at least formally, a weak solution of (1.2) in some region of the $(x, t)$ plane. Moreover we require (1.4) to be satisfied in order to obtain the desired final state.

We wish to solve (2.3) by the method of characteristics (see e.g. [12]).

Let $y \in[1,2]$ be given. Define $\phi_{y}$ the solution (provided it is defined) of

$$
\begin{aligned}
\frac{d}{d t} \phi_{y}(t)= & \alpha a_{\rho}\left(\frac{\phi_{y}(t)+\tau\left(\phi_{y}(t), t\right)}{\alpha}, t\right) \quad \forall t \in[0, T] \\
& \phi_{y}(T)=y .
\end{aligned}
$$

We will also assume that, for any $t \in[0, T], \frac{\phi_{y}(t)+\tau\left(\phi_{y}(t), t\right)}{\alpha}$ lies in the domain

$$
[0, \min (t+\rho, \sqrt{(2-\rho)(t+\rho))}) .
$$

This gives, at least formally, the following formula

$$
\phi_{y}(t)+\tau(y, T)=\frac{t+\rho}{T+\rho}(y+\tau(y, T)) .
$$

In terms of $z_{1}$ this formula is

$$
\phi_{y}(t)=(t-T) z_{1}(y)+y .
$$

At this point of the proof, it is necessary, for the sake of completeness, to distinguish which conditions in our results are required for the state $z_{1}$ for any kind of method of controllability and which are sufficient to apply our technique. 
Since we wish to define the initial data $u(0,$.$) with respect to z_{1}$ we have to require that for two different values of $\left(y_{0}, y_{1}\right) \in[1,2]$ there holds $\phi_{y_{0}}(t) \neq$ $\phi_{y_{1}}(t)$. Thus for any $t \in(0, T]$ we want to have

$$
(t-T) z_{1}\left(y_{0}\right)+y_{0} \neq(t-T) z_{1}\left(y_{1}\right)+y_{1} .
$$

Assume that $y_{0}<y_{1}$ then we must have $(t-T)\left(z_{1}\left(y_{0}\right)-z_{1}\left(y_{1}\right)\right) \neq y_{1}-y_{0}$ or else $\frac{z_{1}\left(y_{0}\right)-z_{1}\left(y_{1}\right)}{y_{0}-y_{1}} \neq \frac{1}{T-t}$; that implies

$$
D^{+} z_{1}(y)=\sup _{h>0} \frac{z_{1}(y+h)-z_{1}(y)}{h} \leq \frac{1}{T}
$$

what we then will assume.

Suppose that equality holds for a point $y \in[1,2]$ such that there exists a sequence $\left(h_{n}\right), h_{n}>0$, for which

$$
\frac{z_{1}\left(y+h_{n}\right)-z_{1}(y)}{h_{n}}>\frac{1}{T} .
$$

Then the backward characteristics issuing from the points $(y, T)$ and $(y+$ $\left.h_{n}, T\right)$ will meet before time $t=0$, thus contradicts the entropic shape of $z_{1}$. Thus the necessary condition is $D^{+} z_{1} \leq \frac{1}{T}$.

Let us stress that this condition is not necessary for $z_{1}$ to be attainable by a general scheme, and relies only on the method introduced here.

Now we have to check that for any $y \in[1,2]$ one has

$$
(t+\rho) z_{1}(y)=\phi_{y}(t)+\tau\left(\phi_{y}(t), t\right) \in[0, \alpha \min (t+\rho, \sqrt{(2-\rho)(t+\rho)})),
$$

which is equivalent to

$$
\forall y \in[1,2], z_{1}(y) \in\left[0, \alpha \frac{\sqrt{2-\rho}}{\sqrt{T+\rho}}\right] .
$$

But this is trivially possible provided one chooses $\alpha$ sufficiently large.

Now we have obtained the conditions announced for $z_{1}$ to be, formally for the moment being, attainable.

Construction of the solution.

Now assume that the condition of Theorem 2.1 are fulfilled. It is obvious that

$$
\Psi:(t, y) \rightarrow\left(t, \phi_{y}(t)=y+(t-T) z_{1}(y)\right)
$$

is a continuous function and that our assumption on uniqueness (2.4) shows that, if $t>0$ is fixed, it is a one-to-one mapping onto its image. Thus the converse map is also continuous and one-to-one (for $t$ fixed). This means that if $t$ is fixed then $y$ depends continuously on $\phi_{y}(t)$. It is also clear that $\Psi([0, T] \times[1,2])$ is the convex set whose boundary is the union of the four intervals

$$
\begin{gathered}
t \rightarrow 1+(t-T) z_{1}(1), t \in[0, T] \\
t \rightarrow 2+(t-T) z_{1}(2), t \in[0, T], \\
{\left[1-T z_{1}(1), 2-T z_{1}(2)\right],} \\
{[1,2] .}
\end{gathered}
$$


We thus are able to define $\tau$ in this region, that is to say $u$, by means of the preceding formulas. This gives also an initial value.

Let us also remark that the initial value may not be continuous, this being the case if $z_{1}$ is a line of slope $\frac{1}{T}$. However, it is of no importance if one uses the following trick.

On the interval $\left[2-T z_{1}(2), 2-\frac{T}{T+\rho} 2\right]$ we define $u(0,$.$) to be the line joining$ the points $\left(2-T z_{1}(2), z_{1}(2)\right)$ and $\left(2-\frac{T}{T+\rho} 2, \frac{2}{T+\rho}\right)$. On the interval $(-\infty, 1-$ $\left.T z_{1}(1)\right)$ we define $u(0,$.$) to be 0$. Now, for the points of $\left[2-T z_{1}(2), 2-\frac{T}{T+\rho} 2\right]$ which are attained by more than one characteristics, we impose to the initial value to have left and right limit at that point. This is possible, because that case occurs when there exists $1 \leq y_{0}<y_{1} \leq 2$ such that $y_{0}-T z_{1}\left(y_{0}\right)=$ $y_{1}-T z_{1}\left(y_{1}\right)$. Now the condition on the derivative of $z_{1}$ and the uniqueness of the curve $t \rightarrow \phi_{y}(t)$ on $(0, T]$ shows that in fact for all $y \in\left[y_{0}, y_{1}\right]$ we have $y-T z_{1}(y)=y_{0}-T z_{1}\left(y_{0}\right)$. Then if $y_{0}=1$ and $y_{1}=2$ then $u(0,$.$) will be 0$ on the left of $y_{0}-T z_{1}\left(y_{0}\right)$ and on the right will be the affine function just as above. If not, let us assume that $y_{0}$ is the infimum value of $y$ such that $y-T z_{1}(y)=y_{1}-T z_{1}\left(y_{1}\right)$ and we redefine $y_{1}$ by the supremum with this property. Then we define

$$
u\left(0, y_{0}-T z_{1}\left(y_{0}\right)-\right)=z_{1}\left(y_{0}\right)
$$

and

$$
u\left(0, y_{0}-T z_{1}\left(y_{0}\right)+\right)=z_{1}\left(y_{1}\right) .
$$

Thus we have defined the initial value, for the Cauchy problem. Moreover this method gives also $u$ by means of $\tau$, following the above manner. We have to check that it is in fact a weak solution to the problem and that it satisfies both final and initial conditions.

We have

$$
\tau\left(\phi_{y}(t), t\right)=\tau\left((t-T) z_{1}(y)+y, t\right)=\tau(y, T) .
$$

Put $x=\phi_{y}(t)$, we get the formula for $t \in(0, T] \tau(x, t)=\tau\left(\xi_{x}^{-1}(t), T\right)$ with $\xi_{x}(t)=y$ if and only if $x=\phi_{y}(t)$. Now it is well known that functions with bounded variations are almost everywhere differentiable. Since $\phi_{y}(t)=$ $y+(t-T) z_{1}(y)$ and that $y \rightarrow \phi_{y}(t)$ is one-to-one onto its image, and because of the assumption on $D^{+} z_{1}$ we immediately see that $\tau$ is almost everywhere differentiable with respect to $x$ and also with respect to $t$, thus it is a weak solution. It is by construction clear that $u$ coincides with $z_{1}$ at times $T$.

Now let us show that for almost every $x \in \mathbb{R}$ one has $\lim _{t \rightarrow 0} u(x, t)=z_{0}$ except for a set of $t$ of measure 0 . Let us make a partition of $\mathbb{R}$ in the following way. Let

$$
\begin{gathered}
I_{1}=\left\{x \in \mathbb{R}, \exists ! y \in[1,2], x=y-T z_{1}(y)\right\}, \\
I_{2}=\left\{x \in \mathbb{R}, \exists\left(y_{1}, y_{2}\right), y_{1} \neq y_{2}, x=y_{1}-T z_{1}\left(y_{1}\right)=y_{2}-T z_{1}\left(y_{2}\right)\right\} .
\end{gathered}
$$

We have

ASSERTION 2.4. $I_{1}$ is a dense union set of open intervals. $I_{2}$ is at most countable. 
Indeed, for any $x \in I_{2}$ there exists a unique maximal interval non reduced to a point, $\left[y_{1, x}, y_{2, x}\right]$ such that for any $y \in\left[y_{1, x}, y_{2, x}\right]$ there holds $x=y-T z_{1}(y)$. Thus since for two different such $x$ the corresponding intervals are distinct, $I_{2}$ is at most countable. It is then clear that $I_{1}$ is of the desired form.

Now let $x \in I_{1}$. Assume that there exists a decreasing sequence of non negative numbers $\left(t_{n}\right)$ such that $t_{n} \rightarrow 0$ and such that $\left(u\left(t_{n}, x\right)\right)$ does not tend to $u(0, x)$. Then we can determine a sequence $\left(y_{n}\right)$ and $y$ in $[1,2]$ such that $u\left(t_{n}, x\right)=u\left(T, y_{n}\right)$ and $u(0, x)=u(T, y)$, but it is also clear that we have $y_{n} \rightarrow y$ (eventually up to a subsequence) so that we have a contradiction.

It is also clear that $u(t, x) \rightarrow u(T, x)=z_{1}(x)$ as $t \rightarrow T$. Thus we have a weak solution of the Burger equation satisfying the desired properties.

Let us remark that the initial data is of bounded variation. Indeed there is a dense union of open intervals on which $u(0,$.$) is of bounded variation. Let$ $\left(x_{n}\right)$ denote the sequence of the points of $I_{2}$. For each $x_{n}$ we can determine two elements of $[1,2]$, say $y_{n}^{1}$ and $y_{n}^{2}$ such that $z_{1}$ is affine on the interval $\left[y_{n}^{1}, y_{n}^{2}\right]$ with slope $\frac{1}{T}$, this interval being as large as possible. Now we have $\left|u\left(0, x_{n}-\right)-u\left(0, x_{n}+\right)\right|=\left|z_{1}\left(y_{n}^{1}\right)-z_{1}\left(y_{n}^{2}\right)\right|$ and thus we see that

$$
\sum_{n \in \mathbb{N}}\left|u\left(0, x_{n}-\right)-u\left(0, x_{n}+\right)\right|<+\infty,
$$

proving that $u(0,$.$) is of bounded variation. Thus we see that in the case$ of a continuous final state, we can obtain a solution to our control problem. This concludes the proof of Proposition 2.3.

2.2.2. Case of more general positive final states. Now we consider the case when $z_{1}$ is no longer continuous. We have.

Proposition 2.5. Assume that $z_{1}$ satisfies the conditions of theorem 2.1 and is $B V([1,2])$ then there exists $u$ satisfying the control problem (1.2), (1.3) et (1.4), with $z_{0 /[1,2]}=0$.

Proof: Let us denote $I_{n}=\left(\alpha_{n}, \beta_{n}\right)$ an open interval on which $z_{1}$ is continuous. By means of the trick used in the proof of the previous proposition, we immediately see that there exists an interval $\left(a_{n}, b_{n}\right)$ with $a_{n}=$ $\alpha_{n}-T z_{1}\left(\alpha_{n}+\right), b_{n}=\beta_{n}-T z_{1}\left(\beta_{n}-\right)$ such that we might define an initial data on $\left(a_{n}, b_{n}\right)$ extended with the same preceding manner producing the desired value $z_{1}$ on the interval $I_{n}$. But here we need to patch all such initial datas defined on such intervals. We use the fact that $z_{1}$ is of entropic shape that is if $y$ is a discontinuity point of $z_{1}$ then there holds

$$
z_{1}(y-)>z_{1}(y+) \text {. }
$$

So if we have a singularity $y$, we determine an interval

$$
\left[y-T z_{1}(y-), y-T z_{1}(y+)\right]
$$

on which we impose $u(0,$.$) to be an affine function joining the point ( y-$ $\left.T z_{1}(y-), z_{1}(y-)\right)$ to the point $\left(y-T z_{1}(y+), z_{1}(y+)\right)$. Now outside the interval $\left[1-T z_{1}(1+), 2-T z_{1}(2-)\right]$ we define $u(0,$.$) just as in the case when$ $z_{1}$ is continuous.

ESAIM: COCV, APRIL 1998, VOL. 3, 83-95 
Finally the same technique as before shows that $u$ is a weak solution to the (1.2) and satisfies both conditions (1.2), (1.3) and (1.4). $\square$

2.2.3. Case of nonnegative final states. In this subsection we give the way when $z_{1}$ is equal to 0 for $x>x_{0}, x_{0} \in(1,2)$ and satisfies also the other conditions.

This is the object of:

Proposition 2.6. Assume that there exists $x_{0} \in(1,2)$ such that $\forall x>x_{0}$ we have $z_{1}(x)=0$ then if $z_{1}$ satisfies the assumptions of Theorem 2.1, there exists $u$ satisfying (1.2) and (1.3) and (1.5).

Proof: For $p \in(1,+\infty), \delta \in\left(0, \frac{1}{2}\right)$ and $\rho \in\left(0, \frac{1}{2}\right)$, let $b_{\delta, \rho, p}$ be as follows: $b_{\delta, \rho, p}$ is the unique entropic solution of (1.2), with the following initial data

$$
\begin{array}{cl}
b_{\delta, \rho, p}(0, x)=\max \left(0, \frac{x}{\rho}\right), & x \leq \rho, \\
b_{\delta, \rho, p}(0, x)=1, & \rho \leq x \leq 1-\delta, \\
b_{\delta, \rho, p}(0, x)=-p, & 1-\delta<x \leq 1, \\
b_{\delta, \rho, p}(x, 0)=0, & \text { else. }
\end{array}
$$

The classical theory of entropic solutions shows that there exist three curves, say,

$$
t \rightarrow x_{1}(t), x_{2}(t), x_{3}(t),
$$

such that for a short time $t \leq t_{1}$ one has (the subscripts are temporarily omitted in $\left.b_{\delta, \rho, p}\right)$

$$
\begin{gathered}
x_{1}(t)=t+\rho, \\
x_{2}(t)=\frac{1-p}{2} t+1-\delta, \\
x_{3}(t)=-p t+1, \\
b(x, t)=\frac{x}{t+\rho}, 0 \leq x \leq x_{1}(t), t \leq t_{1}, \\
b(x, t)=1, x_{1}(t) \leq x \leq x_{2}(t), t \leq t_{1}, \\
b(x, t)=-p, x_{2}(t) \leq x \leq x_{3}(t), \\
b(x, t)=\frac{x-1}{t}, x_{3}(t) \leq x \leq 1 .
\end{gathered}
$$

In fact this holds as long as $x_{1}(t)<x_{2}(t)<x_{3}(t)$.

One has $x_{1}(t)=x_{2}(t) \Longleftrightarrow t=\frac{2(1-\delta-\rho)}{p+1}$ and $x_{2}(t)=x_{3}(t) \Longleftrightarrow t=$ $\frac{2 \delta}{p+1}$. Let us take $\delta<\frac{1-\rho}{2}$, this yields $t_{1}=2 \frac{\delta}{p+1}$.

For $t>t_{1}$, one takes $x_{2}(t)=x_{3}(t)$, and $x_{2}(t)$ is computed for $t>t_{1}$ with the conservation of the mass

$$
\int_{0}^{t+\rho} \frac{x}{t+\rho} d x+\int_{t+\rho}^{x_{2}(t)} 1 d x+\int_{x_{2}(t)}^{1} \frac{x-1}{t} d x=1-\delta(p+1)-\frac{\rho}{2} .
$$

Thus

$$
\begin{aligned}
& x_{2}(t)=t+1-\sqrt{2 \delta t(p+1)} \\
& \text { ESAIM: COCV, APRIL 1998, VoL. 3, 83-95 }
\end{aligned}
$$


as long as $x_{2}(t)>x_{1}(t)=t+\rho$. This holds for $t<\frac{(1-\rho)^{2}}{2 \delta(p+1)}=t_{2}$. Let us point out that $t_{2}>t_{1}$ since

$$
(1-\rho)>2 \delta \text {. }
$$

For $t>t_{2}$, one settles $x_{2}(t)=x_{1}(t)$ and $x_{1}$ is given by

$$
\int_{0}^{x_{1}(t)} \frac{x}{t+\rho} d x+\int_{x_{1}(t)}^{1} \frac{x-1}{t} d x=1-\delta(p+1)-\frac{\rho}{2},
$$

i.e.

$$
x_{1}(t)=\frac{t+\rho-\sqrt{t(t+\rho)} \sqrt{(1-\rho)^{2}+2 \delta \rho(p+1)}}{\rho} .
$$

Of course this is true as long as $x_{1}(t) \leq 1$. For $x_{1}(t) \geq 1$ one has, using again the conservation of mass,

$$
\int_{0}^{x_{1}(t)} \frac{x}{t+\rho} d x=1-\delta(p+1)-\frac{\rho}{2}
$$

i.e.

$$
x_{1}(t)=\sqrt{2(t+\rho)\left(1-\delta(p+1)-\frac{\rho}{2}\right)} .
$$

Let us check, for sake of completeness, that the equality

$$
\frac{t+\rho-\sqrt{t(t+\rho)} \sqrt{(1-\rho)^{2}+2 \delta \rho(p+1)}}{\rho}=\sqrt{2(t+\rho)\left(1-\delta(p+1)-\frac{\rho}{2}\right)}
$$

is possible. This is in fact true for

$$
t_{3}:=t=\frac{(1-\rho)^{2}+2 \delta \rho(p+1)}{2-\rho-2 \delta(p+1)} .
$$

If one fixes $T$, so that $x_{1}(T)=x_{0}$, we need

$$
\left(1-\delta(p+1)-\frac{\rho}{2}\right)=\frac{x_{0}^{2}}{2(T+\rho)}, \text { or else } \delta(p+1)=1-\frac{\rho}{2}-\frac{x_{0}^{2}}{2(T+\rho)} .
$$

This will be possible if $T>t_{3}$.

Now the same method than in the proof of Proposition 2.3 where one replaces $a_{1, \rho}$ by $b_{\rho, \delta, p}$ and choosing $\alpha$ such that

$$
0<z_{1}(x)<\alpha \frac{\sqrt{2\left(1-\delta(p+1)-\frac{\rho}{2}\right.}}{\sqrt{T+\rho}},
$$

gives $z_{1}$ satisfying $z_{1}(x)>\frac{x-1}{T}$, if $x \leq x_{0}$ and $z_{1}(x)=0$ if $x>x_{0}$.

2.2.4. General final state under the assumptions of theorem 2.1. Now we examine the case when $z_{1}$ can take non positive value.

The statement of the main result says that in this case there exists $x_{0} \in$ $(1,2]$ such that, either $x<x_{0}$ and $z_{1}(x)>\frac{x-1}{T}$, either $x>x_{0}$ and $z_{1}(x)<$ $\frac{x-2}{T}$, moreover equality possibly holds when $x=x_{0}$.

We have then:

Proposition 2.7 (Pf. of Theorem 2.1 completed). With the above assumption, one can find $u$ such that the problem (1.2) (1.3) (1.4) has a solution with vanishing initial data on $[1,2]$.

ESAIM: COCV, APRIL 1998, VoL. 3, 83-95 
Proof: For $x \leq 1$, let us define $b_{\delta, \rho, p}(0, x)$ as before and for $x>2$ $b_{\delta^{\prime}, \rho, p^{\prime}}(0, x)=-b_{\delta^{\prime}, \rho, p^{\prime}}\left(0, \frac{3}{2}-x\right)$. Here $p^{\prime}$ and $\delta^{\prime}$ are chosen so that $x_{0}$ is replaced by $3-x_{0}$. Now we are able by the preceding methods to construct an initial data with vanishing value on $[1,2]$ coinciding with $z_{1}$ on $[1,2]$ (in fact at least, almost everywhere). $\square$

\subsection{Case of initial data prescribed on $[1,2]$}

Now we are directly involved in the proof of Theorem 1.1. We thus assume that $u(0,$.$) restricted to [1,2]$ has to be $z_{0}$. We fix real nonnegative numbers, $v, \zeta$ and denote $a_{2, v, \zeta}$ the following data

$$
a_{2, v, \zeta}(x)=\zeta, \text { if } 1-v<x<1 .
$$

We then consider $u^{1}$ the solution of (1.2) with initial value

$$
\begin{gathered}
u^{1}(0, x)=a_{2, v, \zeta}(x) \text { if } x \in(1-v, 1) \\
u^{1}(0, x)=z_{0}(x) \text { if } x \in[1,2] \\
u^{1}(0, x)=0 \text { else. }
\end{gathered}
$$

We will also denote $a_{2, u, \zeta}$ the solution in the entropic class of (1.2) coinciding with its definition at $t=0$. We then have:

AssERTION 2.8. There exists a time $T_{1}$ and $\zeta$ such that the solution of (1.2) $u^{1}$ is $x \rightarrow \frac{x-1+v}{t}$ on $[1,2]$ for $t>T_{1}$.

Proof: We assume a priori that $\zeta>\left|z_{0}\right|_{\infty}$. Let $\mathfrak{a}=\left\|z_{0 /[1,2]}\right\|_{\infty}$. It is then clear that $u^{1}$ is singular at least along a curve, which is Lipschitz denoted $t \rightarrow \xi(t)$ and satisfying

$$
\xi(0)=1
$$

and

$$
\frac{d}{d t} \xi(t) \geq \frac{1}{2}\left(a_{2, v, \zeta}(t, \xi(t)+)-\mathfrak{a}\right), \text { for a.e } t .
$$

Let us remark that this comes from the conservation of the mass of the entropic solutions of (1.2) and can also be interpreted by the characteristic method when the solutions are replaced by their regularization in the $B V$ space.

It will be clear that the claim will be true if one can show that for large enough $\zeta$ the curve $t \rightarrow \xi(t)$ intersects the curve $t \rightarrow$ at +2 in the region $x>1$.

For a short time one has

$$
\text { for a.e. } t>0, \frac{d}{d t} \xi(t) \geq \frac{1}{2}(\zeta-\mathfrak{a}) t .
$$

Thus, for some short time,

$$
\text { for a.e. } t>0 \xi(t) \geq 1+\frac{1}{2}(p-\mathfrak{a}) t
$$

This is available at least up until the following equality holds

$$
\zeta t+1-v=1+\frac{1}{2}(\zeta-\mathfrak{a}) t .
$$


This gives $t=2 \frac{\nu}{\zeta+\mathfrak{a}}$ for which $\xi\left(2 \frac{\nu}{\zeta+\mathfrak{a}}\right) \geq 1+\mu\left(\frac{\zeta-\mathfrak{a}}{\zeta+\mathfrak{a}}\right)$. We thus can only insure afterwards that $\frac{d}{d t} \xi(t) \geq \frac{1}{2}\left(\frac{\xi+v-1}{t}-\mathfrak{a}\right)$, which gives $\xi(t) \geq C \sqrt{t}-$ $v+1-\frac{\mathfrak{a}}{2} t$, with

$$
C \sqrt{2 \frac{v}{\zeta+\mathfrak{a}}}-v+1-\frac{\mathfrak{a} v}{\zeta+\mathfrak{a}} \geq 1+v \frac{\zeta-\mathfrak{a}}{\zeta+\mathfrak{a}} .
$$

We thus obtain

$$
C \geq \sqrt{\frac{\zeta+\mathfrak{a}}{2 v}}\left(v \frac{2 \zeta+\mathfrak{a}}{\zeta+\mathfrak{a}}\right) .
$$

Let us point out that as $p \rightarrow+\infty$ one has $\xi(t) \rightarrow+\infty$. Thus if $\xi(t)>\mathfrak{a} t+2$, for $t>\frac{2 v}{\zeta+\mathfrak{a}}$ we are done.

Let us point out (it will become clearer in the following) that one has to take $\zeta$ as small as possible to obtain an optimal time of control. Let us also point out that this trick becomes unnecessary in the following if one has $z_{0} \geq 0$ on $[1,2]$. Now we choose $\eta>0$ and assume than $T_{1}$ in the preceding claim is chosen so that $\left|u^{1}\right|_{L^{\infty}([1,2])}(t)<\eta$ if $t>T_{1}$. This always is possible because of the asymptotic properties of solutions of $(1.2)$ with finite mass.

We are now ready to give the final proof to theorem 1.1 .

We first of all determine $T_{1}$ as before. We modify $u^{1}\left(T_{1},.\right)$ by imposing that it is 0 outside the interval $[1-v, 2+v]$. For the sake of simplicity let us assume that $z_{1}$ satisfy the condition of Theorem 2.1 . We may modify $b_{\rho, \delta, p}$ in the following way. Let $\mathbb{T}$ be the increasing affine transformation of $[\delta, 1]$ onto $[\delta, 1-v]$. Then we put $b_{\delta, p, p}^{\#}\left(T_{1}, x\right)=b_{\delta, p, p}\left(0, \mathbb{T}^{-1}(x)\right)$ and choose $p$ as in the proof of theorem 2.1, such that the singularity line issuing from $t=T_{1}$ and $x=1-v$ is exactly some $x_{0}$ at the time $T_{1}+T$. We then determine $u\left(T_{1},.\right)$ outside $[1-v, 2]$ such that at the time $T$ the solution $u$ coinciding with $u\left(T_{1},.\right)$ at the time $T_{1}$ is $z_{1}$ on $\left(1, x_{0}\right)$ at the time $T+T_{1}$. This is in fact possible by increasing shortly the value $\alpha$ determined in the proof of theorem 2.1.

Now we glue $u\left(T_{1},.\right)$ with $u^{1}\left(T_{1},.\right)$ and consider the solution $u^{\#}$ of $(1.2)$ with this initial value at the time $t=T_{1}$.

We have:

Assertion 2.9. The solution $u^{\#}$ coincides with $z_{1}$ on $\left(1, x_{0}\right)$ at the time $T+T_{1}$ Moreover one has $\left|u^{\#}\left(T+T_{1}, .\right)-z_{1}(.)\right|_{L^{1}((1,2))}<\eta\left(1+T\left|z_{1}\right|_{L^{\infty}}\right)$.

The proof is immediate. Indeed one has only to see that the singularity line issuing from the point $\left(T_{1}, 1-v\right)$ differs from the one if the initial value on $[1-v, 2]$ was zero at the time $T+T_{1}$ of not more than $\eta T$. Since the final state on this interval is not larger than $\left|z_{1}\right|_{L^{\infty}}$ we have the claim. Then this proves theorem 1.1 , in the case when $z_{1}$ is nonnegative, by taking $v$ small enough.

Now we sketch the case when $z_{1}$ can possibly take negative values.

As in the preceding case one glues $u^{1}\left(t_{1},.\right)$ with the initial value giving $z_{1}$ outside $[1-v, 2+v]$. Following the trajectory of the singularity line issuing from the point shows that it also differs from the one with vanishing initial value at the time $T+T_{1}$ of $\eta T$ we thus have such an equality as in the preceding claim. Here, however we can not insure the coincidence of ESAIM: COCV, APRIL 1998, VOL. 3, 83-95 
the final state with $z_{1}$ on $\left(x_{0}, 2\right)$ but only on $\left(x_{0}+\eta T, 2\right)$ thus we require also that $\eta$ satisfies $\eta(T)<2-x_{0}$. We have then the desired result. This concludes the proof of the main result and the paper.

I specially thank J.-M. Coron for inviting me to treat that problem, and $F$. Ribaud for many indications on the references.

\section{REFERENCES}

[1] F. Ancona, A. Marson: On the Attainable Set for Scalar Nonlinear Conservation Laws with Boundary Control, SIAM J. of control, to appear.

[2] J-M. Coron: Global asymptotic stabilization for controllable systems without drift, Math. Control Signals Systems, 5, 1992, 295-312.

[3] J-M. Coron: Contrôlabilité exacte frontière de l'équation d'Euler des fluides parfaits incompressibles bidimensionnels, C.-R. Acad. Sci. Paris, 317, Série 1, 1993, 271-276.

[4] J-M. Coron: On the controllability of the 2-D incompressible Navier-Stokes equations with the Navier slip boundary conditions, ESAIM: Control, Optimisation and Calculus of Variations, http://www.emath.fr/cocv/, 1, 1996, 35-75.

[5] J.I. Diaz: Sobre la controlabilidad aproximada de problemas no lineales disipativos, proceedings of Jornadas Hispano-Franc sas sobre Control de sistemas distribuidos, A. Valle ed., Univ. de Malága, 1990, 41-48.

[6] C. Fabre, J-P. Puel, E. Zuazua: Contrôlabilité approchée de l'équation de la chaleur semilinaire, C.-R. Acad. Sci. Paris, 315, Série 1, 1992, 807-812.

[7] C. Fabre, J-P. Puel, E. Zuazua: Approximate Controllability of the semilinear heat equation, Proc. of the Royal Soc. of Edinburgh, 125A, 1995, 31-61.

[8] A. Fursikov, O.Yu. Imanuvilov: On controllability of certain systems simulating a fluid flow, IMA vol. in Math. and its Appl. Flow Control, M.D. Gunzburger ed., Springer Verlag, New York, 68, 1994.

[9] A. Fursikov, O.Yu. Imanuvilov: Controllability of evolution equations, Lecture Notes Series 34, Res. Imst., Math. GARC, Seoul National University, 1996.

[10] M. Gisclon: Étude des conditions aux limites pour des systèmes strictement hyperboliques, via l'approximation parabolique, Thèse de l'université Lyon I, 1996.

[11] G. Godlevski, P.A. Raviart: Hyperbolic systems of conservation laws, Ellipses, 1990.

[12] P.D. Lax: Hyperbolic systems of conservation laws and the mathematical theory of shock waves, Regional Conference Series in Applied Mathematics, 11, SIAM: Philadelphia, 1973.

[13] P. Le Floch: Explicit Formula for Scalar Nonlinear Conservation Laws with boundary condition, Math. Methods Appl. Sci., 10, 1988, 265-287.

[14] B.J. Lucier: Regularity through approximation for scalar conservation laws, SIAM J. Math. Anal., 19, 1988, 763-773.

[15] O.A. Oleinik: Discontinuous solutions of nonlinear differential equations, Usp. Math. Nauk (N.S.), 12, 1957, 3-73; English translation: Amer. Math. Soc. Transl., 26, Ser. $2,95-172$.

[16] L. Rosier: Exact Boundary Controllability for the Korteveg-de Vries Equation on a Bounded Domain, ESAIM: Control, Optimisation and Calculus of Variations, http://www.emath.fr/cocv/, 2, 1997, 33-55.

[17] D.L. Russell: Exact boundary value controllability theorems for wave and heat processes in starcomplemented regions, in Differential Games and Control Theory, Roxin, Liu and Sternberg Eds., Marcel Dekker, New York, 1974, 291-319.

[18] A.I. Vol'pert: The spaces BV and quasilinear equations, Math. USSR Sbornik, 2, $1967,225-267$. 